\title{
Assessment of Economic Management of Overlay Traffic: Methodology and Results
}

\author{
Ioanna Papafili ${ }^{1}$, George D. Stamoulis ${ }^{1}$, Rafal Stankiewicz ${ }^{2}$, Simon Oechsner ${ }^{3}$, \\ Konstantin Pussep ${ }^{4}$, Robert Wojcik ${ }^{2}$, Jerzy Domzal $^{2}$, Dirk Staehle ${ }^{3}$, Frank Lehrieder ${ }^{3}$, \\ and Burkhard Stiller ${ }^{5}$ \\ ${ }^{1}$ Athens University of Economics and Business, Athens, Greece \\ ${ }^{2}$ AGH University of Science and Technology, Krakow, Poland \\ ${ }^{3}$ Julius-Maximilian Universität Würzburg, Würzburg, Germany \\ ${ }^{4}$ Technische Universität Darmstadt, Germany \\ ${ }^{5}$ University of Zürich, Zürich, Switzerland
}

\begin{abstract}
Overlay applications generate huge amounts of traffic in the Internet, which determines a problem for Internet Service Providers, since it results in high charges for inter-domain traffic. Traditional traffic management techniques cannot deal successfully with overlay traffic. An incentive-based approach that employs economic concepts and mechanisms is required in order to deal with the overlay traffic in a way that is mutually beneficial for all stakeholders of the Future Internet. This "TripleWin" situation is the target of Economic Traffic Management (ETM). A wide variety of techniques are employed by ETM for optimizing overlay traffic management considering performance requirements of overlay and underlay networks together with cost implications for ISPs. However, the assessment of ETM requires an innovative methodology. In this article this methodology is described and major results are presented as obtained accordingly from the evaluation of different ETM mechanisms.
\end{abstract}

Keywords: Economic Traffic Management; socio-economics; TripleWin; performance; cost; incentives; Internet Service Providers; overlays.

\section{Introduction}

Applications such as peer-to-peer (P2P) file-sharing and video-streaming generate huge volumes of traffic in the Internet due to their high popularity and large size of the files exchanged. This typically underlay-agnostic overlay traffic results in high inter-domain traffic, which implies significant charges for the Internet Service Providers (ISP). Individual optimization in the overlay (decisions made either at random or taking partly into account underlay information) and in the underlay (as in traditional Traffic Engineering) may lead to a sub-optimal situation, e.g., involving traffic oscillations and degraded Quality-of-Experience (QoE) for the end users [1]. Therefore, an incentive-based approach is required that employs economic concepts and mechanisms to deal with the overlay traffic in a way that is incentive compatible for all parties involved, and, thus, leads the system to a situation that is mutually 
beneficial for all end users, overlay providers and ISPs. The so-called "TripleWin" situation is the main target of Economic Traffic Management (ETM) [2] proposed by the SmoothIT project [3]. ETM aims at dealing with the performance requirements of traffic at both overlay and underlay levels, and at reducing ISP inter-connection costs.

SmoothIT has proposed a wide variety of ETM mechanisms aiming at this incentive-based optimization. The entire set of these mechanisms and the synergies identified among them are included in the framework called ETM System (ETMS). ETMS and its particular design choices and implementations offer several alternatives for addressing a selected set of the ALTO (Application-layer Traffic Optimization) requirements [4], formulated in the corresponding IETF working group. Thus, besides providing effective solutions for Internet at present ETM is deemed as applicable to the Future Internet, both conceptually and concerning specific ideas and mechanisms.

All approaches proposed within ETMS are classified in three main categories:

- Locality Promotion enables peers of an ISP domain to receive ratings of their overlay neighbors by an entity called SmoothIT Information Service (SIS). The rating is based on ISP-related factors, such as underlay proximity, and link congestion. An example is locality promotion based on BGP routing data.

- Insertion of Additional Locality-Promoting Peers/Resources involves (a) the insertion of ISP-owned Peers (IoPS) in the overlay or (b) the enhancement of the access rate of Highly Active Peers (HAPS) aiming at both the promotion of locality and faster content distribution. Both approaches, due to the offering of extra capacity resources, exploit the native self-organizing incentive-based mechanisms of overlays to increase the level of traffic locality within ISPs.

- Inter-Domain Collaboration, where collaboration with other domains, either source or destination ones, results in making better local decisions to achieve the aforementioned objectives, due to the extra information made available.

SmoothIT has investigated all of these categories and evaluated them with respect to their performance, reliability, and scalability properties. Here, the focus is laid on the first two categories; note that mechanisms and results discussed here constitute a subset of SmoothIT's investigations. The assessment of ETM requires an innovative methodology (cf. Section 2). The rest of the article is organized as follows: Section 3 deals with the assessment of locality promotion, Section 4 with the insertion of locality-promoting peers/resource, while in Section 5 presents concluding remarks.

\section{Methodology of Assessment}

The detailed studies undertaken to assess ETMS deployment evaluate how all three stakeholders (end users, service providers, and ISP) would benefit thereby and under which circumstances "TripleWin" arises. To attain this, an innovative methodology of assessment for the ETM mechanisms has been developed. The main constituents of this methodology are as follows: 
- The methodology does not consider the optimization of a "total cost" metric for each case; separate objective metrics are used for each player to reflect their diverse requirements and related incentives to employ an ETM mechanism.

- Several evaluation scenarios have been defined to cover a possibility of different ETM deployment degree, popularity of ETM among end users, various swarm sizes, peer distribution among network domains, network topologies etc.

- A game-theoretic analysis is applied to study interactions of ISPs, regarding decisions whether or not to employ an ETM mechanism and the associated ISPs' interactions by taking into account the end user benefit as well.

From the ISP point of view, the ultimate confirmation of "win" is a monetary benefit from ETM deployment. It is, however, not possible to quantify this benefit, since many factors contribute to the overall balance, including deployment and operational costs for an ETM mechanism, savings resulting from inter-domain traffic reduction and the structure of interconnection tariffs, business models, marketing factors. Thus, the assessment methodology focused on another quantifiable metric, namely the inter-domain traffic reduction, since ISPs benefit mostly thereby. In experiments, the inter domain traffic reduction was measured directly (upstream and downstream traffic is evaluated separately) or assessed by a metric called Missed Local Opportunities (MLO). It is a measure of a degree of traffic localization. If a piece of content (e.g., a chunk) is downloaded from a remote domain, although it is available locally, an event of MLO is counted. The less MLOs observed, the better localization is provided by ETM and, thus, an ISP "wins". This metric is used in an ongoing external trial with real users.

As a measure of "win" for end users QoE metrics are used. For file-sharing P2P applications the most important perceivable parameter is download time (or download speed). It can be strongly influenced by ETM mechanisms, both favorably and adversely. Users will use a given mechanism if this improves or, at least, preserves their download time. Ideally, this should be guaranteed on a per individual user basis. However, it is most often analyzed by comparing the average values of the metrics with and without an ETM mechanism. Such averages are taken over all peers in a swarm, or over subsets (e.g., those that belong to the same AS). Main QoE metrics associated with video streaming applications taken into account in assessment of "win" are the probability of playback stalling, stalling time, and start-up delay. These can be influenced by ETM mechanisms as well.

Assessment of "win" for service providers is based on the content availability in the whole overlay (swarm). Another dimension of a service provider's "win" is a decreased traffic volume from its own content servers and reduced load of the servers, as well as an improved performance of the application, which should translate into increased popularity of the service. In reality, these issues often coincide with the objectives of the end users and possibly of the ISPs. Thus, this paper focuses hereafter on assessing win-win for the ISP and end users.

To obtain a reliable assessment of ETM mechanisms several evaluation scenarios have been defined:

- Various network topologies: triangle-shaped, star-shaped, "bike"-shaped, and reflecting a part of the real Internet topology, with a subset of ASes and inter-domain connections; 
- Semi-homogeneous and heterogeneous distribution of peers belonging to a single swarm among ASes; i.e. both "small" and "large" ASes, based on measurements, are considered;

- Varied ETM deployment degree, and interaction between peers located in ASes with ETM deployed and peers located in ASes without ETM;

- Varied end user interest in and adoption of ETM: coexistence of users employing ETM and ones declining support, even within a single swarm;

- Different swarm sizes; and

- Various type of content: files and video of different sizes.

It was assessed whether each player achieves a win, lose, or no-lose situation. It was shown that in certain scenarios a player may benefit or lose depending on whether it implements an ETM mechanism or not, and it was argued that the outcome for a player may depend also on the decisions of other players. The assessment has been carried out by means of simulations. All simulations generate quantitative results for those scenarios considered, but mainly should lead to qualitative results regarding the efficiency of the ETM mechanisms. Nevertheless, certain approximate theoretical models have been defined and investigated numerically, all of which pertain to the simplest evaluation scenarios ([5], [6]). Thus, the attention is directed to simulations.

\section{$3 \quad$ Locality Promotion}

As a selected example for a locality promotion ETM mechanism, the BGP-based one uses the Border Gateway Protocol (BGP) routing information to rate potential overlay neighbors according to their BGP routing distance to the local, querying peer. To this end, metrics like the AS (Autonomous System) hop distance, the local preference value and, if implemented, the MED (Multi Exit Discriminator) value are used. This rating is supported at ETM-enabled clients with mechanisms of Biased Neighbor Selection (BNS) [7] and Biased Unchoking (BU) [8]. The respective mechanism is specified fully and implemented in the ETMS and its client releases. The results from the evaluation of this ETM mechanism have partly already been reported in [8-11].

Here, it shows results from a larger simulation study published in [11]. Specifically, a heterogeneous peer distribution is considered and it is based on live BitTorrent swarm measurements [12], leading to the evaluation of the effect of localityawareness on each of the different user groups separately. This is a new methodology in contrast to related work, where average results or a cumulative density function for all peers is shown, and mainly homogeneous distributions are used.

In SmoothIT's evaluations, a star topology consisting of 20 stub-Autonomous Systems (AS) is applied. Peers and the ETMS servers, providing rating information, are located in these stub-ASes, which are interconnected via a hub-AS containing the initial seed. The access links of peers, which share a file of size $150 \mathrm{MB}$, have a typical ADSL connection capacity of $16 \mathrm{MBit} / \mathrm{s}$ downlink and $1 \mathrm{MBit} / \mathrm{s}$ uplink. The average number of concurrently online peers is between 120 and 200 depending on the scenario, which is a typical swarm size according to the measurements. Peers are distributed hyperbolically from AS 1 to AS 20 according to the distribution in [11], with AS 1 holding the largest share of the swarm, and AS 20 the smallest. 
The SmoothIT client implementations of the locality-aware mechanisms BNS and $\mathrm{BU}$ as described in [8] are applied, comparing the performance of regular BitTorrent [13] with BGP-based locality promotion using both BNS and BU (BGPLoc). For more details about the scenario and the used simulator refer to [11].

In order to assess the performance from an ISP's perspective, the amount of interdomain traffic is considered. In particular, all traffic entering or leaving an AS is considered as inter-AS traffic of the ISP the AS belongs to. This traffic was measured in intervals of one minute during the whole simulation and then averaged over one simulation run. Download times of peers for each AS are presented, mainly to judge the overlay performance from the user's point of view. Here, download times of all peers are averaged in one AS in one simulation run. For each parameter setting 20 simulation runs happened, and the average value over all runs for all observed variables are depicted. The confidence intervals for a confidence level of $95 \%$ are calculated and shown for all scenarios. Fig. 1 and 2 present those results observed.

Fig. 1 outlines that the locality-aware ETM reduces the inter-AS traffic for all ASes, most significantly for the large ASes. Here, it can be stated that a clear win for all providers exist, since they save costs. The amount of these costs depends on the actual agreement between the ISPs and the number of peers of a swarm the ISP holds.

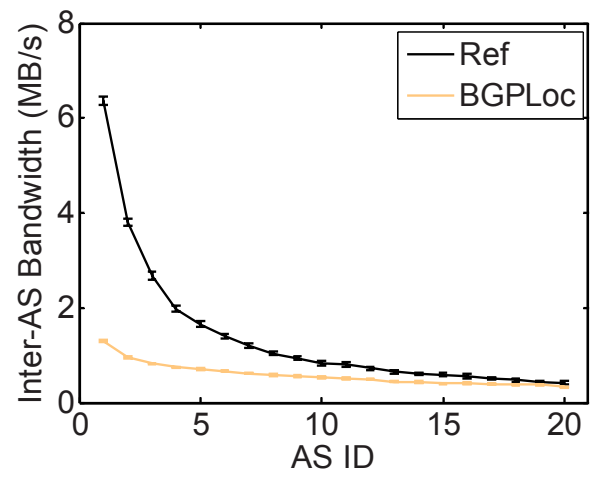

Fig. 1. Mean Inter-AS Bandwidth

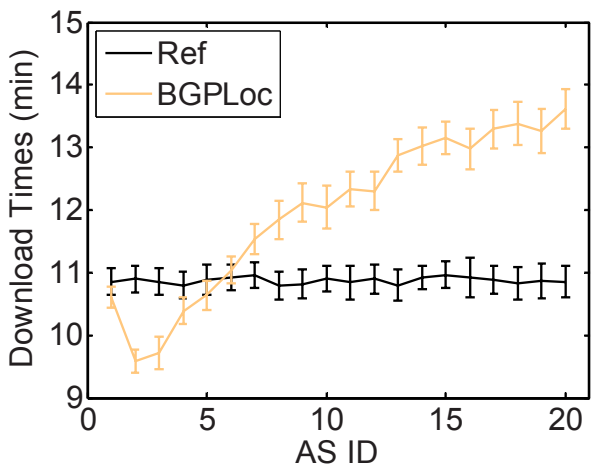

Fig. 2. Download Times

On the other hand, the situation is not as simple when considering end users, cf. Fig. 2. Typically no-lose situations are the result in related work. However, this is true only on average. Due to a shift in upload capacity, which results from applying localityawareness to a heterogeneously distributed swarm, there are large groups of peers that take longer to download the file in comparison to regular BitTorrent. From this perspective, it is not given that users would accept such a mechanism, since they cannot be sure not to lose from it. Furthermore, in another set of experiments investigating the dynamics of BGP-locality adoption, it can be seen that, if large ASes start employing the mechanism one-by-one, smaller ASes will be forced, too. However, to remedy this situation, another mechanism has been developed [11]: smaller ASes are grouped into a meta-AS, with peers in this meta-AS considered as local or at least as closer than the rest of the swarm by all peers in the group. Taking this additional 
mechanism into account, it can be concluded that the locality promotion mechanism in the ETMS may lead to a win-no lose situation, i.e., a reduction in traffic and at least no reduction in the performance for the user, even if considering a more realistic scenario than typically used in related studies.

\section{Insertion of Additional Locality-Promoting Peers/Resources}

The insertion of additional locality-promoting peers/resources implies provision of resources by the ISP in terms of bandwidth and/or storage to increase the level of traffic locality within an ISP, and thus reduce traffic redundancy on its inter-domain links, and to improve performance experienced by the users of peer-to-peer applications. Two approaches, the insertion of ISP-owned Peers (IoPs), and the promotion of Highly Active Peers (HAPs), specifics of methodology employed for their assessment and major qualitative results obtained are described below.

\subsection{Insertion of ISP-Owned Peers}

The ISP-owned Peer (IoP) [14] is a centralized entity equipped with abundant resources, managed and controlled by the ISP. The IoP runs the overlay protocol [13] with minor modifications. It participates actively in the overlay, storing content, and aiming at subsequently seeding this content. Within the seeding phase, the IoP acts as a transparent and non-intercepting cache. As such it can employ existing cache storage management and content replacement policies already used by the ISP. Besides this, the IoP comprises a set of different mechanisms [9] to improve its effectiveness. Extensive performance evaluation has been conducted for all of them [15]. The main focus here is on selected results for the Unchoking Policy and the Swarm Selection. For the latter, a simulation setup with more than one swarm was utilized; this is rarely done in the literature and highlights SmoothIT's assessment methodology.

IoP without and with Unchoking Policy. In the underlay, a simple two AS topology is considered, where the two ASes connect with each other through a hub AS that does not have end users. The tracker and an original seeder are connected to the hub AS, while the IoP is always inserted in AS 1. Values for access bandwidth are similar to ones reported above; only the IoP offers $40 \mathrm{Mbit} / \mathrm{s}$ up and down. In the overlay, a single BitTorrent swarm is considered, with a $150 \mathrm{MB}$ file size and about 120 peers simultaneously online in steady state. Peers arrive according to a Poisson distribution and they serve as seeds for a random time duration that follows the exponential distribution. For further details about the scenario and the simulator refer to [9]. Results are presented as average values over 10 simulation runs along with their corresponding $95 \%$ confidence intervals.

Three cases are evaluated: (1) no IoP insertion (no IoP), (2) IoP, and (3) IoPUP (with Unchoking Policy). Fig. 3 shows high savings on incoming inter-domain traffic of AS1 due to the IoP, but an increase of the outgoing traffic due to the data exchange also with remote peers; however, IoPUP achieves also outgoing traffic reduction 
which could imply monetary savings under more charging schemes compared to the previous case. In Fig. 4, peers' QoE is significantly improved when the IoP is inserted; however, when an IoPUP is inserted, performance is slightly degraded but still improved compared to the no IoP case. Finally, it should be noted that it has been verified (by running couples simulations) that users benefit with respect to performance not just on the average but $95 \%$ of them on an individual basis too.

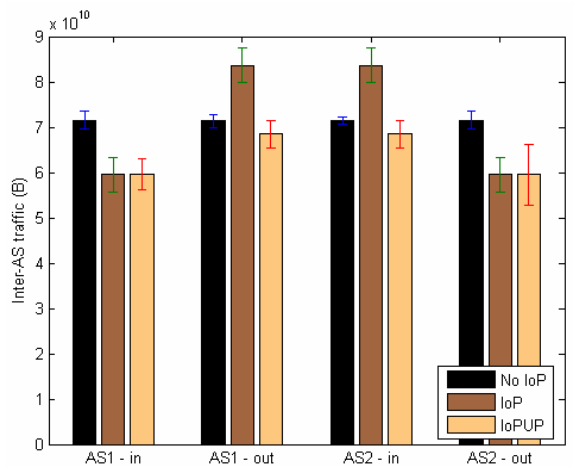

Fig. 3. Mean Inter-AS Bandwidth

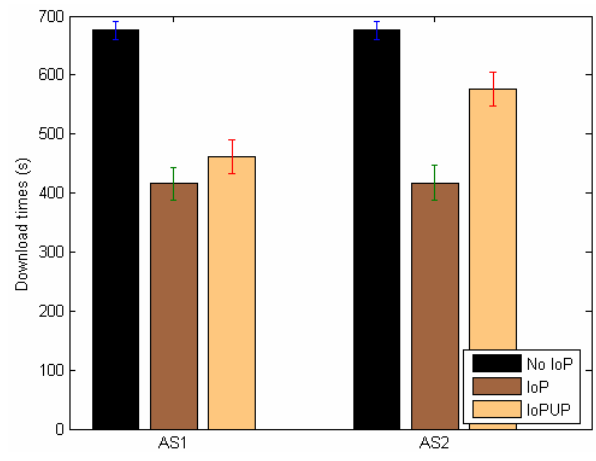

Fig. 4. Download Times

Swarm Selection. In the underlay considered the same setup was applied; only a higher capacity for the IoP, i.e. $50 \mathrm{Mbit} / \mathrm{s}$, is assumed. The overlay assumes two swarms, A and B, that are specified by the three set-up parameters file size, mean inter-arrival time, and mean seeding time. Peers from both swarms exist either in AS 1 , or in AS2, or in both. The default file size is $150 \mathrm{MB}$, the leechers' mean interarrival time is meanIAT $=100 \mathrm{~s}$, and the mean seeding time meanST $=600 \mathrm{~s}$. To study the impact of the three factors, those parameters are tuned only for swarm A as reported in Table 1. For swarm B always default values are employed. For brevity reasons, the case of IoP with policy is not presented here; only results for incoming inter-domain traffic are shown. Further details on this scenario and the simulator are available in [15], [16].

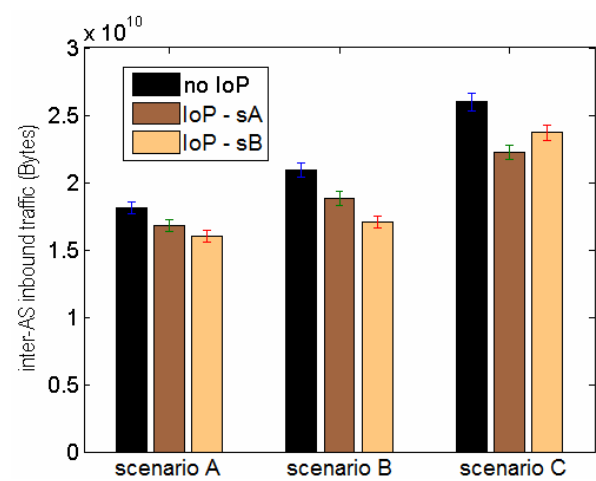

Fig. 5. Mean Inter-AS Bandwidth

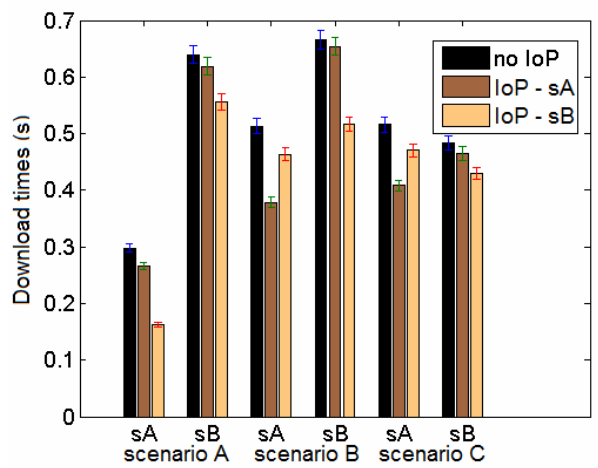

Fig. 6. Download Times 
Table 1. Evaluation Scenarios for the Swarm Selection

\begin{tabular}{c|ccc}
\hline Scenario & $A$ & $B$ & $C$ \\
\hline Modified parameters & File Size: $50 \mathrm{MB}$ & meanIAT: 300.0 s & meanST: 200.0 s \\
\hline
\end{tabular}

Fig. 5 and Fig. 6 present results for the inter-domain traffic and for the peers' download times, respectively. It can be observed that the impact on inbound inter-AS traffic of AS1 is higher when the IoP has joined a swarm with either i) larger file size, or ii) lower mean inter-arrival time, or iii) lower mean seeding time; namely a swarm with higher capacity demand. Performance is always improved since no policy is employed; in each case, higher improvement is observed for the peers of the swarm that the IoP joins. It can be concluded that the IoP provides a win-win situation, if it only uploads locally. Due to the additional upload capacity in the swarm, users benefit from this ETM mechanism, while the inter-AS traffic of the ISP employing the IoP is reduced. However, the efficiency of the mechanism depends on the characteristics of the swarm. Since the IoP can only provide limited resources, the decision about which swarms to join is an important aspect of this ETM mechanism.

A similar issue regarding the Bandwidth Allocation to swarms the IoP has joined has been observed. The effectiveness of this module depends on the number of local leechers and their bandwidth demand, thus, making it important to take this additional metric into account.

\subsection{Promotion of Highly Active Peers}

The Highly Active Peer (HAP) ETM mechanism [17] aims at promoting a more cooperative behavior among overlay peers. If a peer cooperates with the operator by being locality-aware (e.g., by following SIS-recommendations), the operator may increase the upload and download bandwidth of this peer. Additionally, the operator may consider other factors when selecting the peers to promote, such as their contribution to the overlay. In order to provide extra upload and download bandwidth to the peers, the ISP should employ NGN capabilities in its network.

Here, a dynamic HAP operation is considered, which implies instantaneous measurements and an instantaneous reaction of the ISP regarding peer promotion. A static case operates at a longer time range of several hours or even days and was evaluated in a peer-assisted video-on-demand scenario as described in [12].

The main assumption of the HAP ETM mechanism is that by promoting localityaware highly active peers can achieve a win-win situation. The main evaluation goal is to show by simulations that the HAP ETM mechanism allows for decreasing download times of peers that want to become HAPs (due to the extra download bandwidth offered to them). Thus, it is shown that this ETM mechanism provides the right incentive for peers to behave cooperatively, according to ISP goals.

To evaluate the mechanism a triangle topology (three ASes connected with one another) is employed. In each AS, there are 100 peers. AS2 contains 6 initial seeders. The HAP mechanism is deployed only in AS1. The other ASes do not use ETM at all. Further details about the scenario and the used simulator are available in [15]. 
Fig. 7 presents the calculated mean download times of a certain content for the peers in AS1 with respect to the number of HAPs present in the network. The first case, i.e., No SIS is the standard overlay scenario with no ETM mechanisms. '0 HAPs' means that Highly Active Peers were not present; however, the locality promotion mechanism provided by the SIS was used by all peers in AS1. As it can be seen in Fig. 7, the largest difference from the peer point of view is observed if the locality concept is implemented, but do not promote any peer to HAP. Just by introducing the basic locality promotion mechanism, the mean download time decreases significantly (see difference between 'No SIS' and '0 HAPs'); this is due to the fact that peers in AS1 share their resources among themselves, in contrast to the rest of peers, which share their upload capacity with the complete swarm. Afterwards, the download time can be further reduced by increasing the number of active HAPs. This phenomenon can be justified, since each HAP injects more bandwidth to the network, and, therefore, more bandwidth is overall available for peers. This is especially visible when comparing the '100 Peers/AS' with '200 Peers/AS' scenarios (left and right bars, respectively). In the former case, the mean download time is reduced more than in the latter. This is due to the fact that the injection of, say, 20 HAPs increases the available bandwidth by $20 \%$ if the number of peers in the AS is 100 , but only by $10 \%$ when the number of peers in the AS is 200. Therefore, the relative increase in bandwidth is more significant when less peers are present in the AS, hence the difference in download time. The results in Fig. 7 clearly show that end-users benefit from the introduction of HAP ETM mechanism. Not only can they become an HAP and have gain additional download bandwidth, but also, with their extra upload bandwidth HAPs lead to the significant reduction of the average download time too.

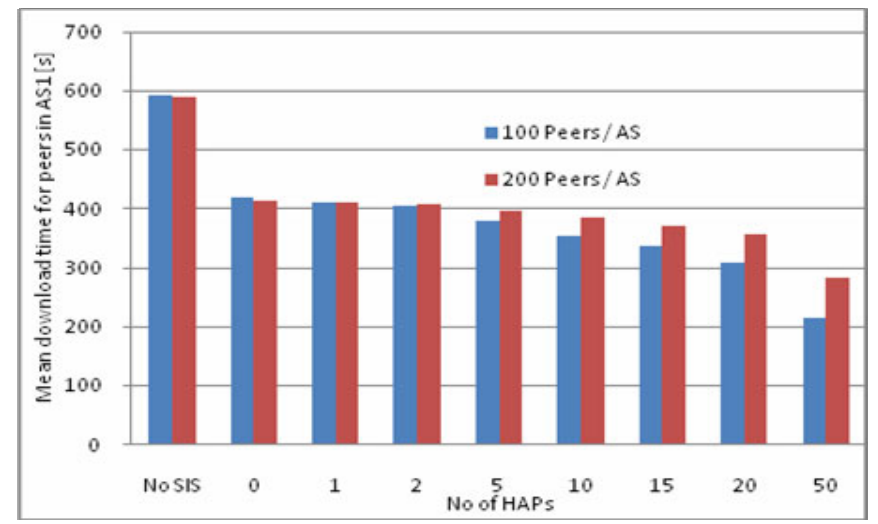

Fig. 7. Mean Download Times for Peers in AS1 with Respect to the Number of HAPs

When an HAP is implemented along with locality-awareness mechanisms, the operator benefits from reduced inter-domain traffic [17]. It allows for reducing costs for ISPs and confirms the advantages of the HAP ETM mechanism and the fact that it achieves win-win. The possibility of becoming an HAP also attracts more peers to use the provided locality mechanisms, therefore, further contributing to the ISP's win. 


\section{Concluding Remarks}

This article presented an innovative methodology of assessment developed within the SmoothIT project especially for the evaluation of a variety of ETM mechanisms and, more specifically, ISP and end users interrelations in the context of such mechanisms. The application of this methodology has been outlined and related evaluation results in three representative mechanisms proposed by SmoothIT have been discussed: (a) the BGPLoc, (b) the insertion of the IoPs, and (c) the promotion of HAPs. Furthermore, this methodology has been employed to assess ETM mechanisms of another category identified, namely the inter-domain collaboration. Moreover, it should be noted that except for static and basic BGP information, an ETM approach can also employ other underlay parameters measured in networks, such as flow throughput, flow delays, and usage of inter- or intra-domain links. Implementation-wise for an operational prototype, the Admin component of the SmoothIT Information Service (SIS) has been designed as a Web-based tool for the ISP to administrate, monitor, and fine-tune the operation of the entire ETMS.

Finally, the extension and application of the methodology for other traffic types (not only P2P) generated according to trends in the Future Internet is an interesting and promising direction for future research.

Acknowledgments. This work has been accomplished in the framework of the EU ICT Project SmoothIT (FP7-2007-ICT-216259). The authors would like to thank all SmoothIT partners for useful discussions on the subject of the paper.

Open Access. This article is distributed under the terms of the Creative Commons Attribution Noncommercial License which permits any noncommercial use, distribution, and reproduction in any medium, provided the original author(s) and source are credited.

\section{References}

1. Liu, Y., Zhang, H., Gong, W., Towsley, D.: On the Interaction Between Overlay and Underlay Routing. In: IEEE INFOCOM 2005, Barcelona, Spain (April 2005)

2. Oechsner, S., Soursos, S., Papafili, I., Hoßfeld, T., Stamoulis, G.D., Stiller, B., Callejo, M.A., Staehle, D.: A framework of economic traffic management employing self-organization overlay mechanisms. In: Hummel, K.A., Sterbenz, J.P.G. (eds.) IWSOS 2008. LNCS, vol. 5343, pp. 84-96. Springer, Heidelberg (2008)

3. SmoothIT Project (December 2010), http://www. smoothit.org

4. Application-Layer Traffic Optimization (ALTO) Requirements: http://tools.ietf. org/html/draft-ietf-alto-reqs-06 (work in progress) (October 2010)

5. Papafili, I., Stamoulis, G.D.: A Markov Model for the Evaluation of Cache Insertion on Peer-to-Peer Performance. In: EuroNF NGI Conference, Paris (June 2010)

6. Lehrieder, F., Dán, G., Hoßfeld, T., Oechsner, S., Singeorzan, V.: The Impact of Caching on BitTorrent-like Peer-to-peer Systems. In: IEEE International Conference on Peer-toPeer Computing P2P 2010, Delft, The Netherlands (August 2010)

7. Bindal, R., Cao, P., Chan, W., Medval, J., Suwala, G., Bates, T., Zhang, A.: Improving Traffic Locality in BitTorrent via Biased Neighbor Selection. In: 26th IEEE International Conference on Distributed Computing Systems, Montreal, Canada (June 2006) 
8. Oechsner, S., Lehrieder, F., Hoßfeld, T., Metzger, F., Pussep, K., Staehle, D.: Pushing the Performance of Biased Neighbor Selection through Biased Unchoking. In: 9th International Conference on Peer-to-Peer Computing (P2P'09), Seattle, USA (September 2009)

9. The SmoothIT Project: Deliverable 2.3 - ETM Models and Components and Theoretical Foundations (Final) (October 2009)

10. Racz, P., Oechsner, S., Lehrieder, F.: BGP-based Locality Promotion for P2P Applications. In: 19th IEEE International Conference on Computer Communications and Networks (ICCCN 2010), Zürich, Switzerland (August 2010)

11. Lehrieder, F., Oechsner, S., Hoßfeld, T., Staehle, D., Despotovic, Z., Kellerer, W., Michel, M.: Mitigating Unfairness in Locality-Aware Peer-to-Peer Networks. International Journal of Network Management (IJNM), Special Issue on Economic Traffic Management (2011)

12. Hoßfeld, T., Lehrieder, F., Hock, D., Oechsner, S., Despotovic, Z., Kellerer, W., Michel, M.: Characterization of BitTorrent Swarms and their Distribution in the Internet, to appear in the Computer Networks (2011)

13. Cohen, B.: Incentives Built Robustness in BitTorrent. In: Kaashoek, M.F., Stoica, I. (eds.) IPTPS 2003. LNCS, vol. 2735, Springer, Heidelberg (2003)

14. Papafili, I., Soursos, S., Stamoulis, G.D.: Improvement of BitTorrent Performance and Inter-domain Traffic by Inserting ISP-owned Peers. In: ICQT'09, Aachen, Germany (May 2009)

15. SmoothIT Project: Deliverable 2.4 - Performance, Reliability, and Scalability Investigations of ETM Mechanisms (August 2010)

16. Papafili, I., Stamoulis, G.D., Lehrieder, F., Kleine, B., Oechsner, S.: Cache Capacity Allocation to Overlay Swarms. In: Bettstetter, C., Gershenson, C. (eds.) IWSOS 2011. LNCS, vol. 6557, Springer, Heidelberg (2011)

17. Pussep, K., Kuleshov, S., Groß, C., Soursos, S.: An Incentive-based Approach to Traffic Management for Peer-to-Peer Overlays. In: 3rd Workshop on Economic Traffic Management (ETM 2010), Amsterdam, The Netherlands (September 2010) 